\title{
FEEDBACK: DIFFERENT CONCEPTS AND PRACTICES IN A CULTURALLY DIFFERENT LEARNING ENVIRONMENT? STUDENTS' PERCEPTIONS
}

\author{
Ahsan Malik, Faiza Kiran*, Fareeha Farooq** \\ Foundation University Islamabad Pakistan, *Army Medical College/National University of Medical Sciences (NUMS) Rawalpindi Pakistan, \\ **Sir Syed College of Medical Sciences for Girls, Clifton, Karachi, Pakistan
}

\begin{abstract}
Objective: To assess students' perceptions of feedback, regarding its understanding, and practices in a traditional undergraduate dental curriculum in Pakistan, where feedback was not part of a formal curriculum as a structured entity.

Study Design: Qualitative-phenomenology.

Place and Duration of Study: Rawal Institute of Health Sciences, Islamabad, from Dec 2017 to May 2018.

Methodology: Purposive sampling was done, and data was gathered using semi-structured, group interview of 16 BDS students. Data was organized using qualitative software package Atlas.ti 8.0 and analyzed using thematic framework analysis. Results: Feedback, though, being practiced by faculty, was not identify as feedback by students. They did not appreciate it as an essential skillset for learning and performance. Though, conceptions of students regarding this phenomenon varied, five emergent themes displayed the essence of the phenomenon in this learning environment: 1) understanding 'feedback' through participants' response, 2) highly variant experiences of information exchange, 3) modes and forms of feedback, 4) factors affecting feedback and 5) methods achieving learning progression.

Conclusion: Our students were devoid of formally instituted feedback practices, so they were unable to identify it as 'feedback'. Wide range of contradictory and many negative experiences of participants indicated that formal training in feedback and its systematic use might improve students' experiences.
\end{abstract}

Keywords: Dental, Feedback, Pakistan, Qualitative, Research, Students, Undergraduate.

How to Cite This Article: Malik A, Kiran F, Farooq F. Feedback: Different Concepts and Practices in a Culturally Different Learning Environment? Students' Perceptions. Pak Armed Forces Med J 2021; 71(5): 1693-1699. doi: https://doi.org/10.51253/pafmj.v71i5.6645

This is an Open Access article distributed under the terms of the Creative Commons Attribution License (https://creativecommons.org/licenses/by-nc/4.0/), which permits unrestricted use, distribution, and reproduction in any medium, provided the original work is properly cited.

\section{INTRODUCTION}

Feedback is an established intervention in medical and dental education to improve learners' performance. ${ }^{1}$ The competency-based education model requires effective feedback skills to develop lifelong learners. In fact, all training programmes where new knowledge and skills are imparted, it must be an essential component. ${ }^{2}$ Feedback can also be used for evaluation of curriculum delivery. Many learning strategies including workshops and group tutorials are also assessed using feedback.

In Pakistan, varying degrees of integration is being practiced in both government and private sector medical colleges. It has replaced traditional large group discussions with modular teaching using small groups utilized for contextual learning. ${ }^{3}$ All these interactive sessions require feedback to assess participants' learning, to gauge delivery of learning objectives and in turn, fulfilment of mission of the institute.

With advancement in medical education, problem-based learning and case based learning has

Correspondence: Dr Ahsan Malik, Assistant Professor HPE, Foundation University Islamabad, Pakistan

Received: 26 Apr 2021; revision received: 24 Sep 2021; accepted: 01 Oct 2021 improved students' clinical reasoning and critical thought process. 4,5 Journal club meetings and presentations have become part of modules as well as faculty development programmes. ${ }^{6}$ All these curricular delivery methods needed a constructive feedback as a vital component of formative assessment, essential for deep learning and personal grooming of learners. ${ }^{7}$

A decade back, in a contextual study, it was anecdotally stated, "In Pakistani medical schools, we usually do not provide our students with feedback which may be restricting the academic development of students as well as the professional development of tutors". 8 The reason might be that the phenomenon be occurring but not identified as 'feedback' amongst stakeholders, who might be unaware of its counterpart in English.

In recent years, foreign students' enrollment in undergraduate healthcare programmes, international health electives and student exchange programmes, ${ }^{9}$ has exposed students to different learning environments with its own set of issues. As a first step to remediation of problems our students encounter, perceptions and practices of feedback need to be assessed. However, few studies in Pakistan have been 
conducted on perceptions of students' feedback, but feedback in dental setting is scant. Therefore, we aimed to explore the concepts and practices of feedback in different modes of instruction in dental colleges of our country. We wanted to highlight the complex nature of feedback interactions in a country with a different learning environment from the west. This unique students' learning environment had a profound effect on their educational outcomes. ${ }^{1}$ Language, religion, culture, beliefs, and political environment of our country also played avital role in forming this learning environment. $^{2}$

The questions aimed in our sdtudy were two-fold. Firstly, what were students' lived experiences of 'feedback' in a traditional dental curriculum in Pakistan?, Secondly, as evidenced through their experiences, how did our students, being devoid of formal, explicit, and systemized feedback mechanisms, achieve the aim of progression of learning. We hope that insights from this study would help to improve curricular delivery and overall health care as feedback in our setting can become an efficient, cost-effective and time saving learning strategy.

\section{METHODOLOGY}

This study was conducted at Rawal Institute of Health Sciences, Islamabad, from December 2017 to May 2018 after ethical approval. We conducted a qualitative phenomenological research using semi-structured group interview. Phenomenology is a form of qualitative research that focuses on the study of an individual's lived experiences within the world. ${ }^{10}$ Being part of a Masters research at the University of Dundee, ethical approval was obtained from University of Dundee School of Medicine Deanery, Research Ethics Committee (CW/sms 23/11/16), while permission to conduct the research was given by the Dental School's management (Principal), as there was no IRB or Ethic Committee in RIHS, at that time.

Sixteen participants were posited to be appropriate to reach the point of saturation in the obtained data. ${ }^{10}$ A purposive sampling was done. Inclusion criteria of participant's selection to ensure maximum variation.

Incluison Criteria: Participants were taken from each year of study (ensuring participation of one from both genders, Urdu and English-medium school environments, residents and boarders).

Exclusion Criteria: First year students were excluded.
In anticipation of our student's apprehensive perception of being 'grilled' for information, we predicted that a group interview would provide a more relaxed environment for sharing of experiences where students would be among their fellow peers. Therefore, a semi structured, group interview of students was conducted which lasted for 40 minutes. Written informed consent was taken by informing participants about nature of the study, their right to withdraw at any time without explanation, no repercussions and assurance of anonymity. A broad interview guide describing areas to be probed was prepared beforehand to guide interviewer in exploring areas of interest from the participants (Figure-1: Appendix A). There were no dropouts of participants in the study.

All participants used Urdu language to varying degrees as a means of expression, mixed with the English language. Though the researcher was fluent in both Urdu and English languages, the rigour of research through second-person review required that bilingual data be translated before analysis. Therefore, Urdu component was transcribed verbatim using phonetic Urdu words in Roman lettering, translated to English and re-translated into Urdu, to obtain the best balance between accuracy of meaning and similarity of original wording.

The audio-taped interviews were transcribed verbatim and analyzed using qualitative software package Atlas.ti 8.0. Themes were identified and principles of reflexivity were applied at each step to ensure rigor of the research outcomes. After the first coding with the use of the coding framework, a second reading of the transcripts alongside the original audio recordings was done to avoid missing substance that was not anticipated while formulating the framework; this strategy aided in preventing the total projection of one's own conceptions onto the participants' experiences. ${ }^{11}$

Categories and themes were compiled on the basis of codes. Going back and forth between data, codes, categories and themes, alignment was ensured among each other and a link within data. The resulting themes were also re-checked with participants to ensure that they correctly reflected the participants' views.

\section{RESULTS}

Interviews of participants resulted in a core interview data of approximately 28000 words. Qualitative analysis of data through thematic framework resulted in the emergence of five themes using quotes by participants. (Figure-2: Appendix B). 
Theme-I: Understanding 'feedback' through study in its conception, methodology and results was participants' response to/use of the term.

no known specific local language (Urdu) term for

A unique factor that significantly shaped our "feedback". This gave rise to the conundrum of how

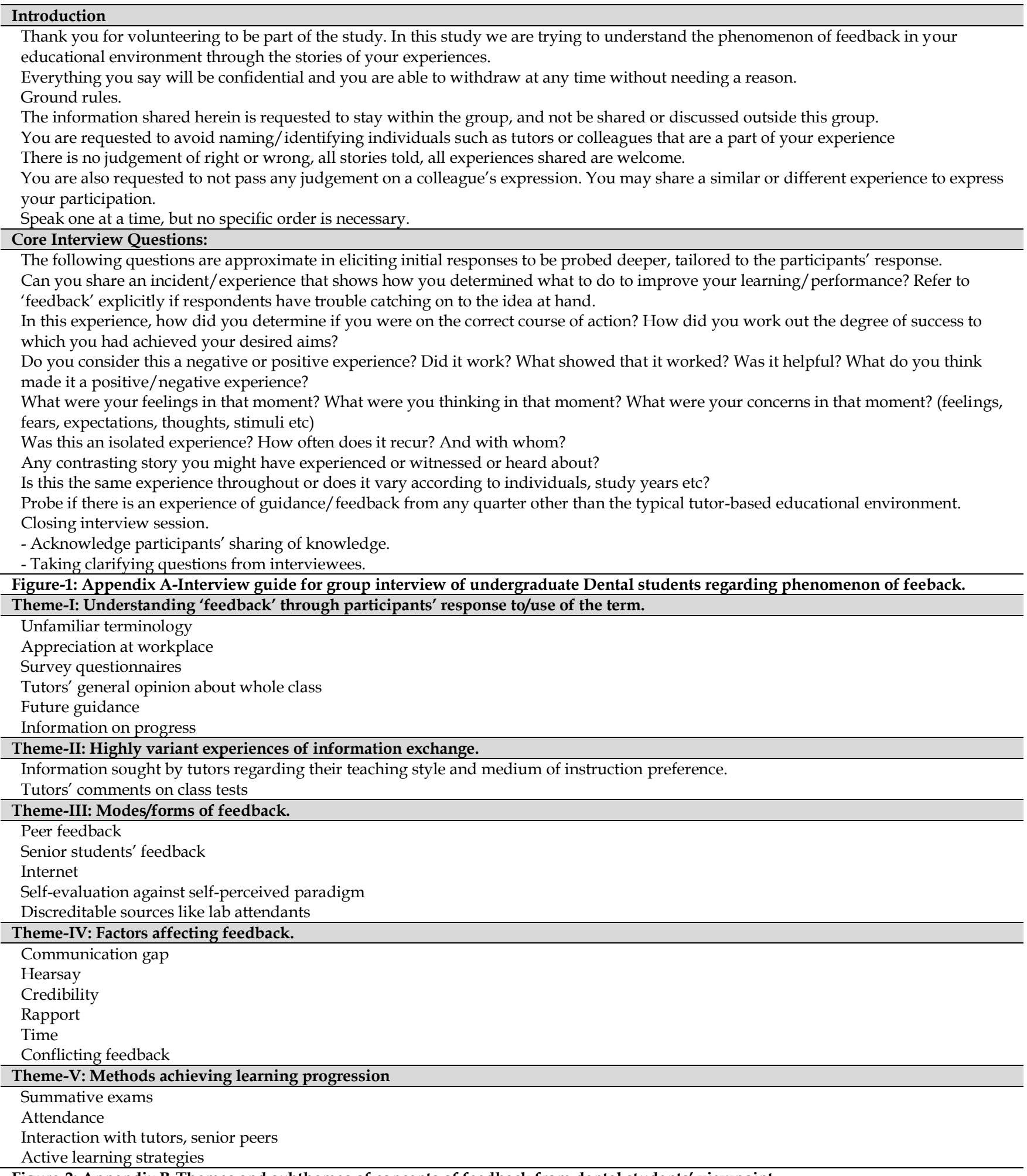

Figure-2: Appendix B-Themes and subthemes of concepts of feedback from dental students' viewpoint. 
to go about exploring this phenomenon through descriptions provided by participants who may be unable to honein on due to unfamiliar terminology.

When participants were asked questions explicitly mentioning the term 'feedback', they seemed not to understand the query. Original question had to be rephrased and explained with examples. When students were prompted to recall incidents in which they gave or received feedback, their responses were centred on completing a questionnaire and judgements about themselves.

A student responded, "Sir we gave that (feedback) for college sports week. It was being asked if extracurricular activities should happen or not. That too was a research of someone from final year from abroad. They gave us pamphlets etc." Another one added, "In clinical work, patients that go from here, they just appreciate a lot. We ask, are you guys satisfied?".

When asked about feedback received from tutors, final year students responded in terms of the response they received at the end of the year from tutors, "After the year completes, (we receive feedback by our teachers in the form of following phrase)' the whole class was very good'. When the year starts (teachers are of the opinion that), 'you are the worst class of the college'.

Second year students responded with conflicting expressions. They were of the view that they did not receive feedback but were given information about the current progress and future direction of their studies, required improvements and guidance about assessments. "Uhh, we don't get much feedback. We get to know this much that if we've previously studied, how much of that we've covered, what we have to study more and how much improvement is needed."

Another response was, "how question in exams comes, we get to know that". Another input was "how to attempt the paper, we get to know that as well."

A student from third year, who came from English-medium school, spontaneously used the term 'feedback' during discussion on clinical laboratory framework. He exclaimed, "the teachers, the house officers, anyone can give their feedback, but they have their own way. 'You shouldn't have made this, too many undercuts, why have you made this in the first place'.

Theme-II: Highly variant experiences of information exchange
The nature of shared feedback experiences amongst students of different years had a lot of variation. Pre-clinical group mentioned tutors seeking information about their teaching styles and teaching 'patterns' so as to change their teaching language or style. One response regarding tutors was, "(They say) If you understand in Urdu then we will speak in Urdu that's not an issue. But the concept is you should understand. Teachers mostly ask us which 'pattern' are you getting English or Urdu? Yesterday's was good or today's".

Same students also expressed how some tutors gave comments on their written work, while some did not despite repeated requests. This was also echoed by students of clinical years. They said, "When we give papers, they give us back, so that we see what our mistakes are. Likewise when we have our vivas and OSPE revisions, we get to know that this is how OSPE is done. (We are told that) this is time management; we have to complete the question in time". Another student added, "sometimes teachers don't show us papers. They just mention list of those who failed or passed". A student reinforced, "it demotivates you and you cannot ask questions about what mistake is done, like how can you improve it." (Other students smiling and nodding).

However, final year students contrasted with students of earlier two years, in that some tutors gave them written work, and the ones that did, they did not receive any additional information except for marked grades. There responses were as follows:

"Other than grade is there anything else on the assignment?"

\section{"Just teacher's sign"}

"I don't think teachers also don't even bother to see. I personally feel that they give marks on basis of names. Like this is that student, give him A plus; ok this is that one, give him B!".

\section{Theme-III: Modes/forms of feedback liked by students}

Students of third year expressed reliance mostly on their peers and seniors. The same degree of reliance was not expressed by students of other years. They were of the following views:

\section{"They're the actual tutors"}

"Friends and internet are most helpful. Well teachers are helpful too but, in the end they're like, we've helped you enough, we've given you questions so just do whatever". 
As the educational years advanced, third-and final year students were more apprehensive about consequences of talking to tutors and institutional authorities about academic issues. Their concerns were, "if it has ever happened once or twice, based on what happened to those students, we have just gone quiet. (So, we are) like, just come, study, do your work, and go".

"They got a Supplee (failed). And they got detained (covers face, all students smiling)".

However, one student from preclinical years said, "yes like those tutors who are new, our HOD asks us and then we tell them that we have this issue."

\section{Theme-IV: Factors affecting feedback}

Various factors shaped the choices of students in giving and receiving information. Teachers being unable to talk at the level of students' comprehension and their negative statements were voiced as few reasons why students hesitated in approaching some tutors. Similarly unreasonable expectations of senior tutors was another cause. "We're so scared to ask questions. We do not ask questions; we just rely on books. The answers that we gave were not up to his (teacher's) standard, and then he's like why you didn't mention this, why didn't you mention that."

Hearsay also played a role in shaping students' behaviour. Final year students mentioned how seniors would scare them away from interacting with certain faculty members, but their experience turned out different. Students shared an incident affronting a male faculty member and a female student, due to which males hesitated in interacting with female students.

"Even now, in final year, we have teachers who hesitate a lot with girls their interaction with boys is good".

"We heard he harassed a student, but our experience with them was good".

"They (senior students) scared us so much from the beginning that this HOD is very strict, but it's not like that".

Perceived credibility and competence of the information source was important for students. Tutors who were seen as less knowledgeable, or had contradictory response to third-party information sources such as books and internet were avoided by students.

"I think sometimes teachers are blank too. Like I remember I asked someone, she was writing calculations on the board and I asked which calculations, and she wrote B.P. calculations right. She didn't reply, she just went to office, came and started writing again".

Clinical group of students also voiced the conflicting nature of feedback received which caused them to fall back to deriving guidance from seniors and peers.

"You make a clasp because some teachers told you how to make it. And another teacher comes and say what is this, this is so wrong. They take it off and lik (hand gestures removing and discarding something). Do it like this.. like this like this I mean you worked a whole day making that thing. And they just come and mess it up for you and then they go their own way".

Rapport between students and tutors affected feedback. Students were more likely to go to those tutors for help who were easily approachable, taught well, explained more than negatively judging them, and with whom they were comfortable.

"Depends who the teacher is. Like HOD of I don't have it in me to go to him again and ask to explain, guide me again. Uhh because he is so strict. Uhh. He keeps saying to us that you come whenever you need, but we know his nature".

The timing of feedback varied; students preferred to have their queries satisfied during lectures, laboratory work and at the end of clinical work, though this did not happen frequently.

"They're not that helpful after lecture. You can ask few questions there, but then again that too is limited. If your concept is still unclear you just can't tell them to stop, or teach it again."

"While you are making a mistake, they're supposed to tell us this is where you're making a mistake. Instead you make the whole denture and after that they tell you to make it again". So the feedback was ineffective as it was not timed right.

\section{Theme- V: Methods achieving learning progression}

Learning was found oriented towards summative exams. Being exam-oriented, students relied on selfstudy and senior peers' guidance. Students preferred interactive learning with tutors where they could freely ask and be responded to instead of passive listening; some students expressed that they would rather not interact with tutors that scared them or made them uncomfortable. "Uhh no not really. We don't treat school exam very seriously. Neither do they. I mean we study for the end of the year exam. the main part of it is at the end of the year". 
The degree to which lectures and tutorials were attended was described as a basic measure of obtaining information about student's attendance, not learning. "If your attendance is good, you're good, if your attendance is bad, you are bad".

"Your whole academic record of the year does not matter. All that matters is your attendance in prof (summative exam) even if their vivas are $100 \%$ even then they are sure to get Supplee (resit).. whose attendance is less. Just on basis of attendance they fail them."

Regarding mode of teaching, there was a wide conflict in choice of tools used by tutors and students.

"Lectures become boring after some time. Teacher is just speaking, rest are sleeping". "If you crack a joke or two, people wake up." Researcher asked, "How many of your lectures go like this?" Students responded, "99 \%" (all nodded).

\section{DISCUSSION}

Students coming from English medium school used the term 'feedback' with greater similarity to how feedback is conceptualized by Ridder et al. ${ }^{12}$ In contrast, students from Urdu medium school described their feedback experiences more in sense of a final (summative) judgement in terms of being good or bad, right or wrong. However, at one instance, students from both schooling backgrounds used the term feedback to refer to how tutors would judge them by failing in summative exams. This suggested that background schooling might not be the only factor at play.

Our study contradicted the assertion of Hamid and Mahmood about Pakistani tutors not providing feedback to students. ${ }^{8}$ This might be due to authors' reference to feedback being confined to the cyclic models of formative feedback, whereas students in our study, associated feedback in sense of information provided as person-centric praise or criticism, summative judgements, and negative criticism of their practical work.

Participants went through a variety of similar and contradicting experiences according to the year of study. For example, second-year students were given marked assignments/tests and were provided with information about their mistakes and how to improve them. Third-year students received verbal comments on year-round assessments, sometimes being denied the opportunity to discuss their performance by few tutors. It can be attributed to lack of training and inc- reased workload on tutors, lack of institutional policy on feedback practices and lack of their expertise ${ }^{13}$. Final-year students expressed not receiving anything other than grades on their assignments. The low utility of these assessment methods may have more than one reason. Students perceived end-of-year exam as the real assessment; they also described the negativity surrounding the aftermath of year-round assessment exercise and difficulties in approaching some tutors which caused them not to take anything on a serious note but the yearly professional exam.

Students labelled tutor markings on textbooks, peer and senior guidance, patients, and examination scores as reliable sources of information about their current performance level. Students also described a reliance on internal feedback (comparison of own performance with an external standard set by themselves) possibly in the face of unfavourable external factors such as unfriendly tutors. Students of clinical years described non-teaching staff such as technicians and clinical assistants as sources of information on their laboratory work, even though they are not authentic sources.

A peculiar mode of feedback described by students was their attendance record. To our knowledge, use of attendance records as an indicator of telling students' performance has not been considered as 'feedback' in any other setting, thus far.

However, all the participants considered a comfortable student-tutor relationship as a necessity for communication that can bring better outcomes. In this regard, there is remarkable similarity in participants' experiences with those described in western-centric studies as well as eastern ones; Harsh and condescending critique has been reported by students as nonconstructive and, therefore, a hindrance to the uptake of feedback. ${ }^{14}$

Another factor affecting feedback practices was time, also reported in other studies in same context. ${ }^{15}$ Students responded to tutor's lack of time for providing sufficiently detailed feedback by turning to other sources such as peers and students of senior years, which may cause inconsistency in feedback. Conflicting feedback was seen in the clinical years as a confounder. ${ }^{16}$ The same was not seen in pre-clinical years. Previous studies have also reported students becoming confused in accepting feedback where subjectivity is involved such as treatment planning of complicated cases and judgement on clinical work done. ${ }^{17}$ 
Hearsay about tutors had affected student-tutor communication to the extent that students avoided those tutors about whom they would hear negative experiences from seniors. At the same time, final year students and some tutors expressed awareness that what they had heard from others was different from their actual experience.

Students had variable notions of the efficacy of learning methods. Lectures and tutorials needed a lot of room for improvement in terms of interaction with tutors in which tutors should tell them about their performance and help them improve upon it.

Assessment driven learning was expressed by students who prioritized studying mainly for summative exams. Other studies in Pakistani context have reported mixed views of students in terms of utility of year-round assessments in which some students were satisfied on receiving only a grade as feedback, while other students wanted more detailed information on their shortcomings. ${ }^{18}$

\section{CONCLUSION}

Informal, unplanned feedback practices were not readily identified by students as "feedback". Students used information from many sources other than tutors for learning progression particularly where feedback was not a formal curricular entity. Wide range of contradictory and negative experiences of participants indicated that training regarding formal feedback and its formative utility might help to streamline students' utilization of feedback in this different learning culture and language.

\section{ACKNOWLEDGEMENT}

Special thanks to Dr Ashley Dennis; the supervisor, for her excellent mentoring.

\section{LIMITATION OF STUDY}

One of the limitations of our study was that data was taken from a single dental institute only, practicing a traditional curriculum. Therefore these findings can only be taken in a specific, contextual, revelatory sense and cannot be applied to the general dental setting in the country, nor to the overall geographical area. However, inclusion of a significant sample from the total population of dental students through a questionnaire, based on insight from the results of our study, may be appropriate for further exploration of the feedback phenomenon.

\section{Disclaimer}

The research was part of the first author's master's dissertation at the University of Dundee, UK (author optedout of publication of dissertation). Highlights of research component were shared in AEME Conference 2018.

Conflict of Interest: None.

\section{Authors' Contribution}

AM: Conceived idea, collected and analyzed data, wrote manuscript, FK: Analyzed data, wrote manuscript, FF: Analyzed data, wrote and edited manuscript.

\section{REFERENCES}

1. Bing-You R, Hayes V, Varaklis $K$, Trowbridge $\mathrm{R}$, Kemp $\mathrm{H}$, McKelvy D, et al. Feedback for learners in medical education: what is known? a scoping review. Acad Med 2017; 92(9): 13461354.

2. Jug R, Jiang XS, Bean SM. Giving and receiving effective feedback: A review article and how-to guide. Arch Pathol Lab Med 2019; 143(2): 244-250.

3. Batool H, Mumtaz A, Chughtai AS. Interactive sessions; lectures and small group discussions: a comparative study in subject of pathology among undergraduate students of central park medical college. Prof Med J 2017; 24(1): 195-199.

4. Dhillon SK, Thaman RG, Sandhu P, Arora DB. Introduction of case-based learning in physiology and evaluation of the learning experience. Pak J Physiol 2018; 12(3): 9-12.

5. Zhao W, He L, Deng W, Zhu J, Su A, Zhang Y, et al. The effectiveness of the combined problem-based learning (PBL) and casebased learning (CBL) teaching method in the clinical practical teaching of thyroid disease. BMC Med Educ. 2020; 20(1): 381-385.

6. Van Diggele C, Burgess A, Mellis C. Journal clubs in health professional practice. Clin Teach 2019; 16(1): 13-18.

7. Ali S, Khan HF. Impact of combined modular assessment on deep learning and personal development of medical students. Pak J Med Sci 2016; 32(1): 191-198.

8. Hamid Y, Mahmood S. Understanding constructive feedback: a commitment between teachers and students for academic and professional development. J Pak Med Assoc 2010; 60(3): 224-227.

9. Button L, Green B, Tengnah C, Johansson I, Baker C. The impact of international placements on nurses' personal and professional lives: literature review. J Adv Nurs 2005; 50(3): 315-324.

10. Gentles SJ, Charles C, Ploeg J, McKibbon KA. Sampling in qualitative research: Insights from an overview of the methods literature. Qualitative Report 2015; 20(11): 1772-1789.

11. Neubauer BE, Witkop CT, Varpio L. How phenomenology can help us learn from the experiences of others. Perspect Med Educ 2019; 8(2): 90-97.

12. Van De Ridder JM, Stokking KM, McGaghie WC, Ten Cate OT. What is feedback in clinical education?. Med Educ 2008; 42(2): 189-197.

13. Gul RB, Tharani A, Lakhani A, Rizvi N F, Ali SK. Teachers' perceptions and practices of written feedback in higher education. World J Educ 2016; 6(3): 10-15.

14. Ansari T, Usmani A. Students perception towards feedback in clinical sciences in an outcome-based integrated curriculum. Pak J Med Sci 2018; 34(3): 702-709.

15. Bhattacharyya H, Vagha J, Medhi GK, Pala S, Chutia H, Bora PJ, et al. Introduction of structured feedback for MBBS students: Perception of students and faculty. J Educ Health Promot 2020; 9(1): 285-290.

16. Al-Hattami AA. The perception of students and faculty staff on the role of constructive feedback. Int J Instr 2019; 12(1): 885-894.

17. Hardavella G, Aamli-Gaagnat A, Saad N, Rousalova I, Sreter KB. How to give and receive feedback effectively. Breathe 2017; 13(4): 327-333.

18. Idrees S, Gul RB, Tharani A, Ali SK. Nurse teachers' conceptions and practices of written feedback in Karachi. J Nurs Educ and Pract 2016; 7(3): 94-98. 